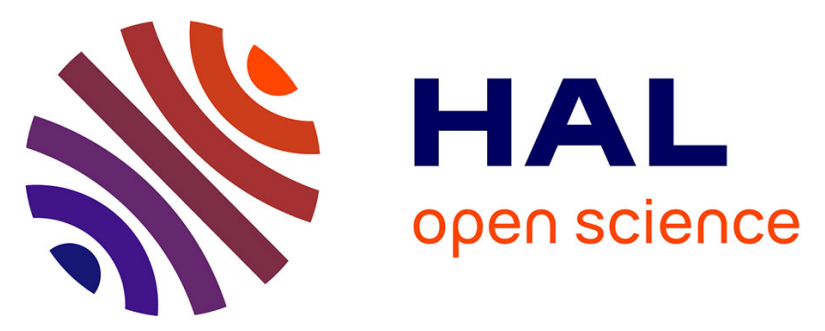

\title{
Validité des informations recueillies auprès des mères sur la période périnatale
}

Pénélope Troude, Laurence Foix L'Helias, Anne-Marie Raison-Boulley, Christine Castel, Christine Pichon, Jean Bouyer, Elise de La Rochebrochard

\section{- To cite this version:}

Pénélope Troude, Laurence Foix L'Helias, Anne-Marie Raison-Boulley, Christine Castel, Christine Pichon, et al.. Validité des informations recueillies auprès des mères sur la période périnatale. Epidemiology and Public Health = Revue d'Epidémiologie et de Santé Publique, 2008, 56 (Suppl. 5), pp.S322. 10.1016/j.respe.2008.06.217 . hal-02423143

\section{HAL Id: hal-02423143 \\ https://hal.science/hal-02423143}

Submitted on 18 Feb 2020

HAL is a multi-disciplinary open access archive for the deposit and dissemination of scientific research documents, whether they are published or not. The documents may come from teaching and research institutions in France or abroad, or from public or private research centers.
L'archive ouverte pluridisciplinaire HAL, est destinée au dépôt et à la diffusion de documents scientifiques de niveau recherche, publiés ou non, émanant des établissements d'enseignement et de recherche français ou étrangers, des laboratoires publics ou privés. 
Publisher's Version/PDF in open access

on editor web site:

https://www.em-consulte.com/showarticlefile/182101/main.pdf

Troude Pénélope, Foix L'Hélias Laurence, Raison-Boulley Anne-Marie, Castel Christine, Pichon Christine, Bouyer Jean, La Rochebrochard Elise (de), 2008, « Validité des informations recueillies auprès des mères sur la période périnatale », Revue d'Epidémiologie et de Santé Publique, 56(Suppl. 5), S322. DOI: 10.1016/j.respe.2008.06.217.

\section{Validité des informations recueillies auprès des mères sur la période périnatale}

Pénélope Troude ${ }^{a, b, c}$, Laurence Foix L’Hélias ${ }^{d, e, f}$, Anne-Marie Raison-Boulley ${ }^{g}$, Christine Castel ${ }^{f}$, Christine Pichon ${ }^{h}$, Jean Bouyer ${ }^{a, b, c}$, Elise de La Rochebrochard ${ }^{a, b, c}$

a Ined, Le Kremlin-Bicêtre, Paris, France

${ }^{b}$ Inserm U822, Le Kremlin-Bicêtre, Paris, France

c Université Paris-Sud, faculté de médecine Paris-Sud, Paris, France

${ }^{d}$ Inserm U149, Villejuif, France

e Université Pierre-et-Marie-Curie - Paris-6, Paris, France

${ }^{f}$ Service de pédiatrie et réanimation néonatales, hôpital universitaire Antoine-Béclère, Clamart, France

${ }^{\mathrm{g}}$ Service de gynécologie-obstétrique, hôpital Foch, Suresnes, France

${ }^{\mathrm{h}}$ Maternité, centre hospitalier intercommunal de Créteil, Créteil, France 
Objectifs. Les caractéristiques périnatales étant liées à la survenue ultérieure d'événements de santé, les études épidémiologiques sur le développement des enfants requièrent des informations sur cette période. En l'absence de registres nationaux, le recueil de données à partir du dossier médical est probablement la source la plus fiable, mais peut s'avérer difficile en pratique, voire impossible. Une alternative est de demander aux mères de remplir un questionnaire postal avec l'aide du carnet de santé de l'enfant. Notre objectif est d'évaluer la concordance entre les dossiers médicaux et les déclarations maternelles concernant les informations sur l'accouchement et la santé de l'enfant à la naissance.

Méthode. Dans trois hôpitaux de la région parisienne, 580 femmes ont rempli un questionnaire postal avec l'aide du carnet de santé de leur enfant, six semaines après leur accouchement. Les informations concernant la grossesse, l'accouchement et la santé de l'enfant à la naissance ont été indépendamment recueillies à partir des dossiers médicaux par les pédiatres de chaque maternité.

Résultats. La concordance entre les deux sources d'information pour différents facteurs périnatals a été évaluée à l'aide de kappas. La concordance était excellente pour le mode d'accouchement, l'âge gestationnel, le poids, la taille et le périmètre crânien de naissance (kappa entre 0,80 et 1,00), bonne pour les hospitalisations durant la grossesse, mais médiocre pour les scores d'Apgar.

Conclusion. À l'exception du score d'Apgar, les déclarations maternelles basées sur le carnet de santé semblent fiables comparées aux dossiers médicaux. Comme le carnet de santé existe dans la plupart des pays développés, cette approche pourrait être utilisée dans les études épidémiologiques sur le développement de l'enfant en France, mais également dans d'autres pays. 